\title{
Ein Thema der problemorientierten Jugendliteratur als Bruch der Tabuzone
}

\section{Jana Baroková}

This article deals with one recent topic of the problem-oriented German juvenile literature - the sexual abuse of children. In the first part of the paper, the literature of the mentioned kind which appeared in the last two decades is characterized and the most attractive topics are shortly described. The following chapter deals with the adolescents as the protagonists of this literature. In the main part of the article, the narrative methods in the novel Gute Nacht, Zuckerpüppchen are analysed in detail. The protagonists and their relationship are presented. The last chapters deal with the topic of sexual abuse in the literary education of children focusing on the preventive role of the children literature at school. Didactic recommendations are added to conclude the discussion.

problem-oriented juvenile literature, break of taboo, sexual abuse, narrative methods, prevention

Dieser Artikel befasst sich mit einem aktuellen Thema der problemorientierten deutschen Kinder- und Jugendliteratur - dem sexuellen Kindesmissbrauch. Zuerst wird die problemorientierte Literatur der letzten zwei Jahrzehnte charakterisiert. Das nächste Kapitel ist den Adoleszenten als Protagonisten der problemorientierten Literatur gewidmet. Den Kern des Artikels bildet die Analyse der narrativen Methoden im Roman Gute Nacht, Zuckerpüppchen. Die handelnden Figuren werden im gegenseitigen Verhältnis präsentiert. Abschließend wird behandelt, wie die Inanspruchnahme von Hilfe in der Handlungsweise der Protagonistin projiziert war. Das letzte Kapitel befasst sich mit der neueren Kinder- und Jugendliteratur zum Thema „Sexueller Missbrauch“ in der literarischen Bildung der jungen Leser.

Problemorientierte deutsche Kinder- und Jugendliteratur, der sexuelle Kindesmissbrauch, Analyse der narrativen Methoden im Roman Gute Nacht, Zuckerpüppchen, literarische Bildung der jungen Leser

Motto:

„Sexueller Missbrauch beschreibt eine Grenzüberschreitung, die immer die Kinder leiden lässt und niemals von ihnen gewollt ist. "

(Bickler 2011: 105)

Im vorliegenden Artikel befasse ich mich anhand des Buches Gute Nacht, Zuckerpüppchen von Heidi Hassenmüller (1941) mit einem heute oft diskutierten Thema: dem sexuellen Kindesmissbrauch. Das Interesse der Medien an diesem ernsten soziologischen Problem ist enorm, die Nachrichten befassen sich damit fast täglich. Die Übergriffe von Erwachsenen auf Kinder finden sich fast in jedem Milieu, sie passieren fast überall, angefangen von kirchlichen Schulen und diversen Erziehungsanstalten und Institutionen bis hin zum Versteck der eigenen Familie. Je heikler und für die psychische Entwicklung des Kindes bedrohlicher das Problem 
wahrgenommen wird, desto mehr wird der Öffentlichkeit bewusst, dass es sich um ein reales Problem handelt, das auch und gerade in der Schule behandelt werden muss, wo die Kinder aufgeklärt werden sollten. Die Lehrer sollten für die Kinder Ansprechpartner sein und mithilfe der Lektüre geeigneter problemorientierter Kinder- und Jugendliteratur mit den Kindern über solche Themen sprechen.

In der Einleitung sollte die Forschungsfrage formuliert werden und der Plan des Artikels vorgestellt werden.

\section{Problemorientierte Jugendliteratur als eine Entwicklungsstufe der antiautoritären Kinderbücher}

Kinder- und Jugendbücher, die sich mit dem sexuellen Missbrauch befassen, gehören zur Gattung der realistischen Kinder- und Jugendliteratur. Für diese Gattung ist charakteristisch, dass in bestimmten Zeiten mit Vorliebe bestimmte Themen behandelt werden. So waren in den siebziger und Anfang der achtziger Jahre Themen beliebt, die Umweltzerstörung, Arbeitslosigkeit oder atomare Katastrophen betreffen, wie z. B. einige Jugendromane von Gudrun Pausewang zeigen.

Seit Ende der achtziger Jahre ist eine starke Verschiebung der Thematik in die innere Welt der handelnden Personen zu beobachten. Neben Themen wie physische Misshandlung, Gewalt in der peer-group, Außenseitertum, Drogenkonsum, Prostitution von Jugendlichen, schwere Krankheit, Behinderung etc. wird in der problemorientierten Jugendliteratur immer öfter und offener das Thema des sexuellen Missbrauchs behandelt. Wenn man die Oberflächenstrukturen wie Figuren, Handlungen, Episoden, Motive und Bilder betrachtet, könnte man zu dem Schluss kommen, dass sich die konkreten jugendliterarischen Werke mit höchst aktuellen Themen befassen. Die Frage ist jedoch, ob die verarbeiteten Themen wirklich jene Phänomene erfassen, die die heutige Jugend betreffen. Um diese Frage zu beantworten, sollten die Tiefenstrukturen der jeweiligen Texte, d. h. die semantischen Grundpositionen des Protagonisten, der Gegenspieler, der Erzählperspektive, aber auch der literarisch dargestellten Zeit, analytisch erfasst werden. Wichtig ist vor allem, wie das Verhältnis des jungen Protagonisten zu den Erwachsenenfiguren, ggf. zu den anderen gleichaltrigen Nebenprotagonisten aussieht (vgl. Gansel 1994: 13-40).

Die problemorientierte Jugendliteratur bemühte sich, lange Zeit als Tabu geltende Themen zu enttabuisieren. Die Helden solcher Werke traten in der Rolle der passiven Opfer auf. Die Enttabuisierung der physischen Übergriffe - eigentlich die Überführung der Täter - geschah wegen des mangelnden Widerstandes nicht durch die gequälten und leidenden Protagonisten selbst, sondern durch ihre agilen Freunde bzw. Mitschüler. So wird die scheinbar auswegslose gewalttätige Familiensituation im Roman Die Sache mit dem Heinrich (1989) von Mira Lobe (1913-1995), wo der Stiefvater den Jungen physisch misshandelt, erst durch das aktive Engagement 
der Mitschülerin Julia gelöst. Dabei scheiterten ihre vorangegangenen Versuche, mit Erwachsenen (seien es Julias Eltern oder ihre Lehrer) über Heinrichs physische Leiden ein offenes Gespräch zu führen, obwohl das Problem allgemein bekannt war. Nach der Beratung mit dem Schuldirektor, der versucht dem Problem auszuweichen, ist selbst die Klassenlehrerin ratlos:

Und haben Sie sich auch gefragt, Frau Kollegin, wie es dann weitergeht? Angenommen, der Vater wird verurteilt, kommt ins Gefängnis, verliert seine Arbeit; der Bub kommt in ein Heim, verliert seine Familie, sein Zuhause. Wem nützt das? Ihrem Schüler Heinrich? Kaum. [...] Wenn ich Ihnen raten darf, Frau Kollegin: Lassen Sie sich die Sache nochmals durch den Kopf gehen ... Und vergessen Sie auch nicht, dass die Swobodas für das Heim zahlen müssten. Es wäre nicht das erste Mal, dass eine Familie durch so eine Anzeige ruiniert wird. (Lobe 1989: 35-36)

Obwohl Julia mehrmals von ihrer Umgebung abgeraten wurde, gelingt es ihr einmal, nach langem Beobachten und nach vielen vergeblichen Versuchen, mit Heinrich Swoboda über seine blauen Flecken und die Schürfwunden auf seinem Körper und Blutverkrustungen im Haar zu sprechen, den Stiefvater beim Gewaltausbruch direkt in der Wohnung der Familie zu ertappen.

Die Helden der problemorientierten Bücher geraten desto mehr in den Teufelskreis der ausweglosen Situation, je weniger ihnen seitens der Erwachsenen - Familie, Lehrer, Erzieher, Vertreter diverser Institutionen - Hilfe geleistet wird und finden sich immer mehr in der Rolle des passiven Opfers. So wird Niklas, der Protagonist des Romans Nicht Chicago, nicht hier (1999) von Kirsten Boie $\left({ }^{*} 1950\right)$ zum Opfer brutaler psychischer Gewalt und materieller Erpressung seitens eines Mitschülers, wobei den Erwachsenen aus dem Umkreis des gequälten Jungen gar nichts auffällt. Kirsten Boie verzichtet auf die Erklärung der Ursachen des Täters sowie auf die Lösung des Konflikts. Umso aktueller wirkt die Botschaft der Geschichte für die Gegenwart. In ihrem Buch stellt Kirsten Boie die Ansicht der Sozialpädagogik in Frage, dass die Eltern mit Hilfe von kompetenten Institutionen - sei es Schule, Sozialamt, Polizei - Probleme lösen bzw. Straftaten verhindern können. Sie wendet sich auch gegen den Grundsatz, dass bei Verhaltensauffälligkeiten der Jugendlichen nach psychologischen Ursachen zu fragen ist (vgl. Schikorsky 2003: 178).

\subsection{Jugendliche als Protagonisten der problemorientierten Literatur}

An dieser Stelle sollten wir uns kurz mit dem psychologischen Terminus „Adoleszenz“ befassen. Die Adoleszenz als eine genau zeitlich begrenzte Phase des Heranwachsens ist nämlich die Voraussetzung für die Entstehung des literaturwissenschaftlichen Begriffs „Adoleszenzroman“ Die folgende Definition bringt meiner Meinung nach den behandelten Terminus treffend zum Ausdruck: 
Die Adoleszenz ist eine lebensgeschichtliche Phase, in der der Zusammenhang zwischen körperlichen, psychischen und sozialen Prozessen besonders deutlich wird. Die sexuellen Reifungsprozesse, die körperliche Möglichkeit zu genitaler Sexualität und dazu, Kinder zu zeugen und gebären zu können, sind der Auslöser für die typischen psychischen und sozialen Entwicklungen während der Adoleszenz: die Ausgestaltung der geschlechtlichen Identität, die Modifizierung des Verhältnisses zu den Eltern und die von ihnen abgegrenzte Gestaltung eigener Liebes- und Arbeitsbeziehungen. (Sauerbaum 1997: 139)

Jugendliche als Hauptfiguren solcher Romane sollen im Folgenden gegenüber den Hauptfiguren in Kinder- und Jugendbüchern anderer Ausrichtung abgegrenzt werden, da diese völlig anders auftreten. Hans-Heino Ewers ${ }^{1}$ charakterisiert die Position des Protagonisten im Adoleszenzroman wie folgt:

In ihm fungiert der Held nicht mehr als Beispielfigur für verallgemeinerbares (Fehl-) Verhalten; er erscheint vielmehr als unvergleichlicher einzelner, der mit seinen ureigenen existenziellen Problemen im Zentrum der Darstellung steht. Individualisierung und Psychologisierung kennzeichnen denn auch diese Erzählgattung. (Ewers 1996: 14)

\subsection{Beziehung der Eltern zu den Kindern und vice versa in der problemorientierten Jugend- und Adoleszenzliteratur}

In der Jugend- und Adoleszenzliteratur wird im betrachteten Zeitabschnitt der letzten fünf Jahrzehnte eine ganze Skala von Beziehungen der Jugendlichen zu den Eltern als sozialer Einheit bzw. den Müttern und Vätern präsentiert. Seit Anfang der 70er Jahre verändert sich mit der Entstehung der literarischen Phase der sog. „Anderen Moderne“ in der Kinder- und Jugendliteratur und mit der Ablehnung der Darstellung einer heilen Welt zugunsten der Welt, in der Kinder an den sozialen, zwischenmenschlichen, gesundheitlichen und anderen Sorgen der Erwachsenen beteiligt sind, die Darstellung der Sozialeinheit Familie. Die Familie ist nicht mehr eine harmonische Idylle des familiären Zusammenlebens.

Im Zuge der Verwandlung der traditionellen Geschlechterrollen in den 80er und 90er Jahren verändert sich das Schema der Rollenaufteilung des früheren klassischen Familienmodells. Die Konstellation des Zusammenlebens variiert nicht nur infolge der gestiegenen Zahl der Ehescheidungen, sondern auch infolge der steigenden Attraktivität von neuen Formen des Zusammenlebens, verursacht durch die ökonomische Unabhängigkeit der Frauen von den Männern. Es entstehen Familien mit allein erziehenden Elternteilen, Wochenendfamilien, Fortsetzungsfamilien, Wohngemeinschaften bis hin zu den sog. Patchwork-Familien (vgl. Wild 1996: 56-67). Unkonventionelle Familien kreieren in ihrem jugendliterarischen Werk

1 Hans-Heino Ewers ist Professor für Germanistik/Literaturwissenschaft mit dem Schwerpunkt Kinder- und Jugendliteratur an der Johann Wolfgang Goethe-Universität Frankfurt/Main. Seit 1990 ist er Direktor für Jugendbuchforschung. 
z. B. die österreichischen Autorinnen Christine Nöstlinger ${ }^{1}$, Barbara Frischmuth ${ }^{2}$ oder Jutta Treiber ${ }^{3}$. Die moderne Jugendforschung stellt im Vergleich zur vorangegangenen Generation entscheidende Veränderungen der Familienstruktur fest. Die Eltern treten allmählich immer weniger in der Rolle der Erzieher auf und werden zu älteren Freunden der Kinder (siehe Mädchenromane der oben genannten österreichischen Autorinnen). Sie sind stärker zur gegenseitigen Verständigung mit den Kindern bereit, und die Kinder werden als gleichberechtigte Gesprächspartner angenommen. Aufgrund dieses Emanzipierungsprozesses wächst natürlich das Selbstbewusstsein der Kinder.

\section{Analyse der narrativen Methoden im Roman Gute Nacht, Zuckerpüppchen von Heidi Hassenmüller ${ }^{4}$}

Der mit dem jugendliterarischen Preis „Buxtehuder Bulle“5 ausgezeichnete Mädchenroman Gute Nacht, Zuckerpüppchen mit dem Thema des sexuellen Kindesmissbrauchs schöpft aus den autobiographischen Kindheitserlebnissen der Autorin Heidi Hasenmüller selbst. Ihre auf dem Umschlag abgedruckten Worte drücken prägnant die Dringlichkeit des Themas aus: „Es ist die Geschichte meiner Jugend, die ich nicht hatte ... Ich habe sie für all die Mädchen geschrieben, die in der gleichen auswegslosen Situation sind. Durchbrecht das Schweigen und ruft um Hilfe. Immer wieder, bis man euch hört“" (Hassenmüller 1992: Umschlagseite).

Die Geschichte spielt in den ersten Nachkriegjahren in Hamburg, die nicht nur durch materielle Knappheit, sondern auch durch die traumatischen Auswirkungen des Zweiten Weltkrieges geprägt sind. In Gabys Familie fehlt der Vater, der im Zweiten Weltkrieg gefallen ist. Der künftige Stiefvater der Kinder erscheint eines Tages unerwartet und mit größter Selbstverständlichkeit an der Schwelle der Wohnung. Die Ankunft des unbekannten Mannes, der sich als angeblichen ehemaligen Freund des Vaters der Kinder ausgibt, enthält die explizit warnende Symbolik des Märchens Der Wolf und die sieben Geißlein. Die Lektüre der Passage lässt den Leser gleich am Anfang des Buches die Gefahr, die den Geschwistern Gaby und Achim droht, miterleben.

1 Vgl. Jugendromane von Christine Nöstlinger (*1936) Man nennt mich Ameisenbär, Ilse Janda, 14, Gretchen Sackmeier - Eine Familiengeschichte, Der Zwerg im Kopf.

2 Vgl. den Jugendroman Ferienfamilie von Barbara Frischmuth $\left({ }^{*} 1941\right)$.

3 Vgl. den Jugendroman Solange die Zikaden schlafen von Jutta Treiber $\left({ }^{*} 1949\right)$.

4 Heidi Hassenmüller ist 1941 in Hamburg geboren. In den Niederlanden, wo sie seit vielen Jahren lebt, studierte sie Journalistik und Belletristik. Heute schreibt sie für verschiedene Tageszeitungen und Zeitschriften. Sie hat bereits mehrere Jugendbücher veröffentlicht.

5 Der jugendliterarische Preis „Buxtehuder Bulle“ wurde 1971 von der Buchhandlung Ziemann \& Ziemann gestiftet und wird seither jährlich für das jeweils beste Jugendbuch aus der laufenden Produktion vergeben. Es wird durch eine Jury von 11 Erwachsenen und 11 Jugendlichen vergeben. Die Abstimmung und Entscheidung erfolgt nach einem Punktsystem ohne vorherige Absprache, um die Spontanität der Entscheidung vor allem bei den jugendlichen Juroren nicht zu beeinflussen. 
Es klingelte. Die Kinder zählten: einmal, zweimal, dreimal. [...] „Wer ist da?“

Du musst immer fragen, wer da ist, bevor du die Tür aufmachst, hatte Mutti ihr eingeschärft. Gaby begriff das. Sie dachte an den Wolf und die sieben Geißlein. Die waren gefressen worden, weil sie den Wolf hereingelassen hatten. [...] „Ich möchte zu Frau Mangold“, rief ein Mann. Frau Mangold war Mutti, und Mutti schlief, weil sie nachts lange arbeitete. [...] „Mach bitte auf“, rief der Mann. „Ich bin ein Kriegskamerad deines Vaters.“ (Hassenmüller 1992: 6-7)

Die historische Kulisse der Handlung bildet die Gegenwartsgeschichte. Konkret sind dies die ersten Jahre nach dem Zweiten Weltkrieg. Obwohl das Thema des Kindesmissbrauchs in den Medien heute oft besprochen wird und die Öffentlichkeit dadurch immer mehr beunruhigt wird, gelingt es der Autorin mit der Rückkehr der Erzählung in ihre eigene Kindheit, also in die von der Gegenwart nicht weit entfernte Vergangenheit, beim Leser den Eindruck zu erwecken, dass es sich beim Problem des sexuellen Kindesmissbrauchs um ein zeitlich entferntes, für die Gegenwart nicht mehr so aktuelles Problem handelt, was natürlich nicht der Wahrheit entspricht. Im Hinblick auf die Protagonistin Gaby, ihr durch Angst, Scham und Besorgnis um das Leben der Mutter gelähmtes Handeln, kann beim Leser der Eindruck entstehen, dass sich Mädchen von heute - also emanzipierte und selbstbewusste moderne Protagonistinnen - anders, d. h. aktiv und laut wehren würden. Es ist interessant der Frage nachzugehen, inwieweit sich die im Werk abgebildete Realität der vierziger und fünfziger Jahre ins Handeln und Denken der Protagonistin und der weiteren handelnden Personen projiziert und inwieweit Jugendliche von heute anders handeln würden.

Die im Buch dargestellte materielle Knappheit begleitet den Alltag der vaterlosen Familie. Die Authentizität der totalen Armut, vor allem des Mangels an Lebensmitteln in den ersten Nachkriegsjahren, zeigt meiner Meinung nach am besten ein Satz vom Anfang des Buches: „Gaby kaute mahlend auf der Brotrinde. Sie war hart, aber mit viel Spucke wurde aus der harten Kruste ein Brotbrei“ (Hassenmüller 1992: 5). Der Verlockung des von Anton Malsch - so heißt der unbekannte Ankömmling angebotenen Schokoladeriegels kann Gaby nicht widerstehen und wehrt massive Vorwürfe ihres Bruders Achim schwach ab, dass sie sich nur wegen Süßigkeiten gegenüber dem fremden Mann freundlich verhält. Während sich die anfänglich gegenseitige Missachtung zwischen Achim und Onkel Anton - wie der fremde Mann auf Wunsch der Mutter von den Kindern genannt werden soll - zu einer offenen Feindschaft zuspitzt, verwandelt sich Gabys anfängliche naiv-kindliche Bewunderung für den Stiefvater in eine tiefe Zuneigung. Anton Malsch handelt nämlich planmäßig und zielbewusst als heimtückischer Täter, um sein primäres Ziel zu erreichen: Gaby zum Objekt seiner sexuellen Übergriffe zu machen. Die spießbürgerliche Moral der Nachbarn der Familie setzt sich in einer schnellen Heirat der Mutter mit Anton Malsch durch, was paradoxerweise für dessen 
Pläne von Nutzen ist. Der schwache Einfluss der Sozialbehörden als angeblichen Vertreter und Beschützer der Interessen der Kinder erschöpft sich in einer einzigen vagen Bemerkung - gemeint quasi als eine versteckte Rüge - eines Beamten am Vormundschaftsgericht: „In einem Zimmer mit Herrn Malsch und nebenan schlafen Ihre Kinder" (Hassenmüller 1992: 12).

Die regelmäßigen und immer brutaler werdenden sexuellen Übergriffe des Stiefvaters auf Gaby gehören für die Protagonistin allmählich zum schauderhaften Alltag. Das Mutter-Tochter Verhältnis wird durch das patriarchalische Modell der Familie als einer vollkommen vom Mann abhängigen sozialen Einheit stark beeinflusst. Die Tatsache, dass Anton Malsch die Kinder wegen der Halbwaisenrente nicht adoptiert hat, erklärt die Mutter den Kindern dadurch, dass sie durch die Adoption ihren Rentenanspruch verlieren würden. Aufgrund des völligen Fehlens jeder Art von Emanzipation sieht die Mutter am Verhalten ihres Mannes gegenüber Gaby nichts Beunruhigendes. Der Leser kann sogar der Eindruck gewinnen, dass sie vor der schrecklichen Realität systematisch die Augen verschließt. Aufgrund der auswegslosen Situation gerät Gaby in einen Teufelskreis, den nicht einmal die Lehrerin, Fräulein Moll, bzw. der Hausarzt imstande sind zu durchbrechen, obwohl Gaby mehrmals Andeutungen macht, wie z. B. mit der Ablehnung des Familiennamens des Stiefvaters:

„Ihr lebt allein bei eurer Mutter?“, fragte Fräulein Moll.

Gaby errötete: „Nein, meine Mutter hat wieder geheiratet. Ihr Mann lebt auch bei uns.“ $[\ldots]$

„Das ist dann dein Stiefvater.“

„Ja“, Gaby räusperte sich. „Aber mein Bruder und ich heißen noch immer Mangold.“

(Hassenmüller 1992: 40)

Die Protagonistin sucht panisch, jedoch total introvertiert und immer tiefer in eine krankhafte psychische Einsamkeit versinkend, die Rettung und zieht sich in ihrer Phantasiewelt und ihren Erinnerungen absolut zu ihrem leiblich abwesenden Vater zurück. Nur selten gelingt es ihr, verzweifelte Signale in ihre reale Umgebung zu senden, die jedoch für die zuständigen Erwachsenen zu vage Umrisse haben, als dass diese etwas begreifen könnten, wie es z. B. das Gespräch mit dem Hausarzt zeigt:

Dr. Rehbein ging zurück zu seinem Schreibtisch und machte sich ein paar Notizen. „Ich werde mit deiner Mutter und deinem Vater sprechen."

„Ich muss gehen, meine Mutter wartet.“ An der Tür drehte Gaby sich noch einmal um. „Übrigens, mein Vater ist tot.“ (Hassenmüller 1992: 39)

Den physischen und psychischen Verfall, den Gaby aufgrund des sexuellen Missbrauchs durchlebt, bezeichnet Sigrid Steinbrecher zutreffend als „Vaterfalle“ (vgl. Steinbrecher 1991). Die Figur des toten leiblichen Vaters wächst in der Innenwelt der Protagonistin zum unerreichbaren Sehnsuchtsobjekt, obwohl 
seine Gestalt auf der narrativen Ebene nur marginal bleibt. An dieser Stelle ist zu bemerken, dass der Einfluss von Vätern auf die heranwachsenden Kinder in der patriarchalischen Gesellschaft grundsätzlich idealisiert wurde, wobei den Müttern eindeutig eine passive und angepasste Rolle zugeschrieben wurde.

Die Autorin Heidi Hassenmüller lässt ihre Protagonistin aufgrund ihrer Erlebnisse wortwörtlich pure Höllenqualen erleben. Das befriedigende Resultat der Erlösung für Gaby ist nur als ob scheinbar stark auf die sachgemäße Substanz des Satzes des Hausarztes Dr. Rehbein am Ende des Buches komprimiert formuliert. Die Aussage Gabys Mutter beinhaltet dagegen die Verzweiflung einer Frau, die nicht mutig genug ist, der nackten Wahrheit in die Augen zu sehen:

Dr. Rehbein sah starr zu Mutti., Wenn Sie nicht unterschreiben, sorge ich dafür, dass Ihr Mann ins Gefängnis kommt. Nicht nur wegen Misshandlung.“ [...] „Wie soll ich denn weiterleben, wenn ich das glauben würde, was Sie sagen? Dann könnte ich mich ja gleich aufhängen.“ [...] „Es dreht sich jetzt nicht darum, wie Sie weiterleben. Es dreht sich darum, dass Gaby weiterlebt. Und dazu braucht sie Ihre Unterschrift." (Hassenmüller 1992: 134)

\subsection{Handlungsstruktur}

Der Handlungsverlauf ist in drei nicht proportional große Teile gegliedert. Im einführenden Teil wird die dreiköpfige Familie - Mutter, Sohn und Tochter - als die heile Welt vorgestellt, wobei der fremde Ankömmling sich als ein alter Freund des im Krieg gefallenen Vaters der Kinder vorstellt. Der Hauptteil besteht aus einem langen Handlungsstrang, dessen Ereignisse (v. a. der sexuelle Missbrauch von Gaby) chronologisch ablaufen, wodurch der Leser allmählich den Eindruck gewinnt, dass es keinen Ausweg aus der Situation des missbrauchten Opfers gibt. Im Schlussteil erfährt der Leser von der Befreiung des Opfers durch den behandelnden Hausarzt. Der weitere zukünftige Lebensweg der Protagonistin wird auktorial sachlich kommentiert, die psychische Entspannung der Heldin bleibt allerdings auf expliziter Ebene aus. Die verwendeten Zeitebenen verdeutlichen die andauernde, stetig wachsende Verzweiflung der Protagonistin. Während sowohl der Einführungsals auch der Schlussteil knapp und bündig präsentiert werden, wirkt die Zeit der sexuellen Misshandlung - es handelt sich dabei nur um ein paar Jahre - auf den Leser wie eine Hölle ohne Ende.

Die Figurenkonstellation ändert sich im Laufe der Handlung wie folgt: Der Sohn wird wegen des bereits offenen Hasses auf den Stiefvater von der Familie ausgeschlossen; die Tochter zieht sich während der Zeit des sexuellen Missbrauchs immer mehr in sich zurück; die Mutter schließlich schlüpft in eine immer passivere und dem Mann untergeordnetere Rolle. Die Erzählperspektive ist die des 
auktorialen Erzählers, die Gefühle und das Leid des Mädchens werden objektiv sachlich kommentiert und so erfährt der Leser mit der Zeit die Veränderung der Protagonistin von einem fröhlichen Mädchen zu einer verstörten, schüchternen, in sich zurückgezogenen Jugendlichen. Die Sprache des Textes entwickelt sich von einer anfänglich metaphorischen Darstellung der erotischen Berührungen hin zur brutalen, eindeutigen Schilderung des Geschlechtsverkehrs. Die Ohnmacht, Angst und Schamgefühle des Opfers werden realistisch, jedoch sprachlich sehr sparsam ausgedrückt.

\subsection{Opfer des sexuellen Missbrauchs}

Welche tiefen Spuren der sexuelle Missbrauch an Gaby hinterlassen hat, erfährt der Leser allmählich, im Zuge der Schilderung der zunehmend inadäquaten Reaktionen der Protagonistin auf die Erwachsenen, sei es der Hausarzt, der Gynäkologe oder die Lehrerin. Auch ihre immer stärker werdenden Schuldgefühle gegenüber der Mutter und sogar gegenüber dem Stiefvater stimmen mit denen von anderen missbrauchten Kindern überein. Da die Geschichte zu einem Zeitpunkt beginnt, an dem Gaby noch nicht missbraucht wurde, also noch in einer "heilen Welt“ lebte, wird zwar sehr kurz, aber dennoch über ihren Charakter vor dem Missbrauch erzählt. Der gefallene Vater dient dem Mädchen und ihrem Bruder als Vorbild und ist für sie das Ideal eines Helden und gleichzeitig eines liebevollen Vaters. Gaby spürt alle negativen Folgen eines missbrauchten Kindes: Es kommt zu psychosomatischen Reaktionen bei dem Verdrängen der negativen Erlebnisse. Durch das Idealisieren des Vaters in ihrer Phantasie versucht sie, sich der schrecklichen Realität zu entziehen. In ihre soziale Umgebung - Ärzte, Erzieher, Lehrer - sendet sie zwar scheinbar schroffe, jedoch eindeutige Hilferufe aus, die nur langsam begriffen bzw. durch die Mutter verdrängt werden.

\subsection{Täter}

Anton Malsch, Gabys späterer Stiefvater, entspricht in allen Zügen seines Charakters dem Tätertypus, dem die Neigung zur sexuellen Misshandlung zugetraut werden kann. Dies wird von außen z. B. durch sein vorgetäuschtes Freundschaftsbündnis zum toten Vater der Kinder, durch seinen Dilettantismus im Beruf, als auch durch die Untreue gegenüber seiner Ehefrau im Roman angedeutet. Im Handeln in der Familie und vor allem gegenüber Gaby geht er zielbewusst und unerschrocken als planmäßiger Täter vor. Er verwendet gegen Gaby nicht nur physische sondern auch psychische Gewalt und schreckt nicht einmal vor der psychischen und existenziellen Erpressung seiner Frau zurück. Sowohl vor als auch nach der Vertreibung des gehassten Stiefsohnes aus dem Haushalt meint er, er sei unverletzlich und seine Macht uneingeschränkt. 


\subsection{Opfer-Täter-Verhältnis}

Das Buch schildert den Missbrauch des Stiefvaters an seiner Stieftochter, also die sexuellen Übergriffe im familiären Nahbereich. Die Personenkonstellation ist realistisch, da die Konstellation Täter, also ein Mann innerhalb der Familie, und ein Mädchen, also die Stieftochter, beim sexuellen Missbrauch häufig vorkommen. Typisch ist auch die anfängliche Zuneigung und Sympathie des Mädchens gegenüber dem Stiefvater, weil dadurch die Gefühlslücke, die nach dem Tod des Vaters zurückgeblieben ist, gefüllt werden soll. Gaby ist also am Anfang besonders empfänglich für die Berührungen des Stiefvaters, obwohl sie seitens des älteren Bruders massiv kritisiert wird. Auf seine kategorisch ablehnenden und verachtenden Vorwürfe reagiert sie ihrem Alter entsprechend. Anton Malsch verwendet bei seinen Taten zuerst immer intensiver werdende Berührungen von Körperteilen, bis er sich zum Geschlechtsverkehr vorwagt. Während er Gaby bei den anfänglichen Berührungen ihres Körpers durch Vortäuschung von Zuneigung und „väterlicher" Liebe desensibilisiert, schreckt er nach dem schließlich vollzogenen Geschlechtsverkehr mit ihr nicht vor Erpressung und Bestechung zurück, bis er schließlich brutale physische Gewalt ausübt, die letztlich die Institutionen wie Pädagogen, Erzieher und Ärzte auf den Plan rufen und zum Einschreiten veranlassen.

\subsection{Inanspruchnahme von Hilfe}

Im mündlichen Kontakt mit der Lehrerin bzw. mit dem Arzt wehrt sich Gaby massiv gegen die Bezeichnung „dein Vater“ seitens der Vertreter der Institutionen (siehe oben die Inhaltsanalyse des Werkes). Dieses Verhalten ließe sich zwar als Hilferuf interpretieren, wird von den Adressaten aber nicht als solcher wahrgenommen, weil es zu wenig kategorisch ist. Bei der Unterstützung von Gaby spielt nicht die passive und psychisch labile Mutter, die aufgrund ihres Selbstmordversuchs um sich eine feste Barriere der Unberührbarkeit aufgebaut hat, eine Rolle, sondern die schlagfertige Freundin von Gaby, zusammen mit ihrer Mutter, mit der Anton Malsch ebenfalls seine pathologischen Praktiken auszuüben wagte. Die Aufdeckung der sexuellen Gewalt, die Anton Malsch jahrelang an seiner Stieftochter begangen hat, folgt sehr schnell; präsentiert wird sie jedoch nur anhand des Gesprächs des behandelnden Arztes mit Gabys Mutter (siehe oben die Inhaltsanalyse des Werkes). Das Thema der Inanspruchnahme von Hilfe wird im analysierten Werk so dargestellt, dass der Leser zum Hilferuf ermutigt wird. Im vorliegenden Werk ergibt sich die Ermutigung aus dem Zusammenhang der Handlung, wo der Leser die demütigenden und brutalen Übergriffe von Anton Malsch und die psychische und physische Qual Gabys sehr langwierig und desto nachdringlicher empfindet und so zum Hilferuf motiviert wird. 


\section{Die neuere Kinder- und Jugendliteratur zum Thema "Sexueller

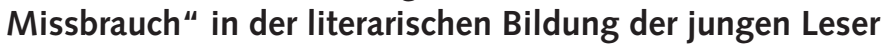

Die Leser sehen sich bei der Lektüre der Bücher zum Thema „Sexueller Missbrauch“ mit einer realen und problematischen Lebenswirklichkeit konfrontiert, wobei die verwendete personale Erzählform der Autoren von ihnen eine emotionale Beteiligung fordert. Trotz der ernsten Probleme gelingt es Heidi Hassenmüller, den jungen Rezipienten eine optimistische Weltsicht zu vermitteln, da die Protagonisten die Chance bekommen, ihr Leben in die eigene Hand zu nehmen. Die am Ende der 1960er Jahre erhobene Forderung, sich in der Jugendliteratur mit den Fragen der Erziehung und des sozialen Verhaltens zu beschäftigen, führte dazu, dass die Realität ohne Beschönigung als ein für Erwachsene und Kinder gemeinsamer Erfahrungsraum dargestellt wurde. In diesem Rahmen wurde Kinder- und Jugendliteratur zum Gegenstand des Literaturunterrichts. Antiautoritäre Kinderbücher leiteten Ende der 70er Jahre eine Trendwende ein, die eine deutliche Erneuerung der Kinderliteratur zur Folge hatte. Durch die antiautoritäre Kinderliteratur wurde erstmals das vorher in der Kinder- und Jugendliteratur dominante heile Familienbild in Frage gestellt. Das ganze Erzählrepertoire wurde erweitert, thematische Tabus wurden gebrochen und es wurde offen auf soziale Konflikte hingewiesen. Wenn als Ausgangspunkt für das Begegnen mit Literatur die aktuelle Thematik dient - d.h. Themen wie Scheidung und Trennung, Liebe und Hass, Armut und Existenzprobleme - lassen sich gerade realistische Kinder- und Jugendbücher gut in der Schule einsetzen.

Der sexuelle Kindesmissbrauch wird in der Kinder- und Jugendliteratur bereits im frühen 20. Jahrhundert thematisiert. In den Aufklärungsbüchern trat ab 1933 der bedrohliche „schwarze Mann“ auf, der den Mädchen Böses - nicht näher beschrieben - antun konnte. Später wurde es üblich, Kinder zu warnen: „Geh nie mit einem Fremden mit“. Eine viel größere Aussagekraft für junge Leser haben authentische Aussagen der betroffenen Opfer, die in den Medien bzw. in der Literatur über ihre Situation sprechen, was auch der Fall von Heidi Hassenmüller und ihrem Werk ist. Die Wirksamkeit eines Buches ist dann am größten, wenn es die Gefühlswelt und den Verstand der Kinder trifft. Erst unter diesen Umständen kann das Werk zur Prävention eingesetzt werden (vgl. Bickler 2011: $34 \mathrm{ff}$ ).

\subsection{Rolle der Kinderliteratur bei der Prävention in der Schule}

Nicht nur in der Schule, sondern bereits im Kindergarten und in der Vorschule werden Materialen zur Prävention von sexuellem Missbrauch entwickelt - Puppen, Spiele, Ausmal- und Bilderbücher etc. -, die im Unterricht zu diesem Thema eingesetzt werden können. Wenn man die Kinderbücher für Vorschulkinder bzw. die bis zur 3. Klasse, die das Thema behandeln, betrachtet, so sind eindeutig 
diejenigen nicht zu akzeptieren, die den sexuellen Missbrauch nicht klar benennen, sondern nur andeuten, dass etwas Schreckliches passiert ist und dem Leser so im Unklaren lassen, worum es sich eigentlich handelt. Die Qualität der Kinderbücher sollte bewertet werden, ob folgende Fragen untersucht werden:

- Wird das Opfer des sexuellen Missbrauchs charakterisiert?

- Wird der Täter näher dargestellt?

- Handelt es sich um physische und psychische Gewalttaten? Wie war ihre Dauer?

- Inanspruchnahme von Hilfe

- Wie wurde die sexuelle Gewalt aufgedeckt? (vgl. Bickler 2011: 38).

Sowohl in Kinder- als auch in Jugendbüchern kann sexuelle Gewalt thematisiert werden. In Jugendbüchern ist die Darstellung aber im Allgemeinen ausführlicher und direkter als in Kinderbüchern. Der im Rahmen der Sexualerziehung thematisierte Kontext befasst sich also erst ab dem Alter von 10 Jahren mit sexuellen Rechten. Es wird über Verantwortlichkeit und über Sexualität gegen den eigenen Willen informiert. In den Jugendbüchern wird anhand der Handlung beispielhaft gezeigt, wie man sich gegen den sexuellen Missbrauch wehren und Hilfe holen kann. Gleichzeitig werden auch misslungenen Hilferufe thematisiert. Karin Bickler unterscheidet in diesem Zusammenhang drei Typen solcher Jugendbücher: Jugendbücher des ersten Typs enthalten Texte, die aufgrund der eigenen Lebenserfahrungen des Autors entstanden sind. Das oben analysierte Werk Gute Nacht, Zuckerpüppchen von Heidi Hassenmüller (1992) ist ein Beispiel für diesen Buchtyp. Bücher des zweiten Typs befassen sich mit den Folgen der Qual der Protagonisten. Bücher der dritten Gruppe befassen sich sowohl mit dem sexuellen Missbrauch innerhalb der Familie als auch mit der emotionalen Misshandlung.

\subsection{Didaktische Empfehlungen}

Problemorientierte Kinder- und Jugendbücher eignen sich sehr gut für den Unterricht, weil sie die Kinder zur Auseinandersetzung mit existenziellen Fragen anregen und Lösungsmodelle auf dem Gebiet der Prävention, der Diagnostik und der Therapie altersgemäß anbieten. Es gibt drei Einsatzfelder, wo sie behandelt werden können: Erstens ist dies der Unterricht, der auf literarische Bildung zielt, zweitens ist dies die Leseförderung und drittens der themen- und problemorientierte Unterricht (vgl. Hurrelmann 2003: 146. In: Bickler 2011: 94). Anhand der Lektüre bekommen die Kinder eine bessere Chance, die Strategien der Täter zu durchschauen und die angebotenen Lösungsmodelle ggf. im eigenen Leben anzuwenden. Für die Jugendlichen haben diese Bücher eher einen sekundären Präventionscharakter. Es werden vor allem die Tragweite und die Folgen von sexuellen Übergriffen auf das künftige Leben verdeutlicht. 
Man muss jedoch auch mögliche negative Folgen der Lektüre solcher Bücher berücksichtigen. Sie können bei Kindern und Jugendlichen u. U. Angstgefühle erzeugen. Es muss daher in den Texten deutlich erkennbar sein, dass für die Gewalttaten die handelnden Erwachsenen verantwortlich sind, nicht die Kinder und Jugendlichen. Ein weiteres Problem bringt Darstellung der Folgen mit sich. In vielen Büchern begegnen die Kinder rational handelnden Erwachsenen, die immer sofort eine Lösung finden. In der Realität glauben aber die Erwachsenen den Kindern oft nicht, wenn sie von ihren Leiden berichten. Auch kann es in der Realität schwierig sein, geeignete Gegenmaßnahmen zu treffen. Die Behandlung von sexuellem Missbrauch im Unterricht erfordert einen sachlichen, altersgerechten und vertrauensvollen Kontext. Auf dem Gebiet des literarischen Lernens, das einerseits zur Persönlichkeitsentwicklung und anderseits zur literarisch-ästhetischen Bildung beitragen soll, ergibt sich neben Unterrichtsgesprächen vor allem die reflexiv-analytische Auseinandersetzung mit dem Text. Hier haben die Schüler die Möglichkeit - hinter den Protagonisten des behandelten Buches versteckt - von sich selbst nicht sprechen müssen. Bei den Kindern soll auch die Leseförderung, also das Bedürfnis die Bücher selbständig in der Freizeit zu lesen, entwickelt werden. Gerade aber bei der Problematik des sexuellen Missbrauchs ist vor der „freien Lesezeit“ ohne Besprechen in der Schule abzuraten. Darüber hinaus können solche Bücher aber auch in anderen Unterrichtsfächern gut angewendet werden wie z. B. in der Mensch-, Natur- und Kulturkunde bzw. in der Familien- und Sexualerziehung.

\section{Fazit:}

Anhand des analysierten Buches habe ich auch mithilfe einiger anderen Werke gezeigt, dass in der deutsch geschriebenen problemorientierten Jugendliteratur ab 1990 dem Leser immer öfter ein tiefer Einblick in die Psyche von jugendlichen Opfern sexueller Gewalt gewährt wird. Dieser Aspekt ermöglicht solche Bücher im Unterricht einzusetzen und sie mit den Schülern zu diskutieren. Im behandelten Mädchenroman Gute Nacht, Zuckerpüppchen wird durch ein authentisches Kindheitserlebnis der Autorin Heidi Hassenmüller eine schwer benennbare Realität in Worte gefasst, wodurch Identifikationsmöglichkeiten angeboten werden, die aus einer ganz neuen Sichtweise Handlungsmuster zeigen und Lösungswege anbieten. Die erste und wichtigste Voraussetzung dafür ist jedoch, Tabus zu durchbrechen und sich mit der Missbrauchsthematik aktiv auseinanderzusetzen. Meines Erachtens ist es der Autorin mit der Schilderung ihrer eigenen, sich in den 50er Jahren des 20. Jahrhunderts abspielenden Kindheitserlebnisse gut gelungen, den zeitgenössischen potentiellen kindlichen Opfern des sexuellen Missbrauchs zu zeigen, dass das vorhandene Problem enttabuisiert und rechtzeitig gelöst werden kann. 


\section{Literaturverzeichnis}

\section{Primärliteratur:}

Boie, Kirsten (1999): Nicht Chicago. Nicht hier. Hamburg, Oetinger Verlag.

Frischmuth, Barbara (1981): Die Ferienfamilie. Salzburg und Wien, Residenz Verlag.

Hassenmüller, Heidi (1992): Gute Nacht, Zuckerpüppchen. Berlin, Rowohlt Taschenbuch Verlag.

Lobe, Mira (1989): Die Sache mit dem Heinrich. Wien, München, Verlag Jungbrunnen.

Nöstlinger, Christine (1974): Ilse Janda - 14. Hamburg, Oetinger Verlag.

Nöstlinger, Christine (1986): Man nennt mich Ameisenbär. Wien, Oetinger Verlag.

Nöstlinger, Christine (2012): Der Zwerg im Kopf. Weinheim, Beltz und Gelberg Verlag.

Nöstlinger, Christine (1981): Gretchen Sackmeier. Hamburg, Oetinger Verlag.

Treiber, Jutta (1998): Solange die Zikaden schlafen. Wien, Ueberreuter Verlag.

\section{Sekundärliteratur:}

Bickler, Katrin (2011): Sexueller Missbrauch als Thema der neueren Kinder-und Jugendliteratur. Freiburg im Breisgau, Herbert-Jürgen Welke Fillibach Verlag.

Daubert, Hannelore (1997): Von „jugendlichen Eltern und „erwachsenen“ Jugendlichen. Familienstrukturen und Geschlechterrollen in Schülerromanen der 80er und 90er Jahre. In: Doderer Klaus / Ewers, Hans-Heino (Hg.): Jugendkultur im Adoleszenzroman. Weinheim und München, Juventa Verlag. S. 43-61.

Gansel, Carsten (1997): Jugendliteratur und jugendkultureller Wandel. In: Doderer Klaus / Ewers, Hans-Heino (Hg.): Jugendkultur im Adoleszenzroman. Weinheim und München, Juventa Verlag. S. 15-18.

Ewers, Hans-Heino (1997): „Was geht in deren Köpfen vor?“ Zur jugendliterarischen Auseinandersetzung mit dem jugendlichen Rechtsextremismus. In: Ewers, Hans-Heino (Hg.) Jugend- und Adoleszenzroman 4/1996. Leipzig, Ernst Klett Schulbuchverlag. S. 7-18.

Hurrelmann, Bettina (2003): Kinder- und Jugendbücher im Unterricht. In: Grundzüge der Literaturdidaktik. Hrsg. Klaus-Michael Bogdal, Hermann Korte. 2. Aufl. München 2003, S. 134 - 146. In: Bickler, Katrin (2011): Sexueller Missbrauch als Thema der neueren Kinder- und Jugendliteratur. Freiburg im Breisgau, Herbert-Jürgen Welke Fillibach Verlag. S. 94.

Moransee, Jess R. (1982) (Hg.): Preisgekrönte Kinderbücher. Ein internationales Verzeichnis von 193 Kinderbuchpreisen. München, K.G. Saur Verlag. S. 225.

Sauerbaum, Evelyn (1997): Literarische Erkundungen weiblicher Adoleszenz in aktuellen Jugendbuchproduktionen. In: Doderer Klaus / Ewers, Hans-Heino (Hg.): Jugendkultur im Adoleszenzroman. Weinheim und München, Juventa Verlag. S. 139-164.

Schikorsky, Isa (2003): Kinder-und Jugendliteratur. Köln, Literatur und Kunst Verlag.

Wild, Inge (1996): Vater - Mutter - Kind. In: Ewers, Hans-Heino (Hg.) Jugend- und Adoleszenzroman 4/1996. Leipzig, Ernst Klett Schulbuchverlag. S. 56-67.

Steinbrecher, Sigrid (1991): Die Vaterfalle. Die Macht der Väter über die Gefühle der Töchter. In: Sauerbaum, Evelyn (1997): Literarische Erkundungen weiblicher Adoleszenz in aktuellen Jugendbuchproduktionen. In: Doderer Klaus / Ewers, Hans-Heino (Hg.): Jugendkultur im Adoleszenzroman. Weinheim und München, Juventa Verlag. S. 139-164.

PhDr. Jana Baroková, Ph.D.

Katedra německého jazyka a literatury

Pedagogická fakulta MU, Brno 